\title{
Sudan National AIDS Spending Assessment Rounds: A Systematized Review
}

\author{
M. Kheir Sara Gabrallah ${ }^{1}$, Habbani Khalid ${ }^{2}$ and Mousnad MA* ${ }^{3 *}$ \\ ${ }^{1}$ University of Medical Sciences and Technology (UMST), Sudan \\ ${ }^{2}$ Alsudani Center for Training in Health Economics, Sudan \\ ${ }^{3}$ Health Economic Centre, University of Khartoum, Sudan
}

*Corresponding author: Mohamed Awad Mousnad, Ph. D, RPh, Consultant of Pharmacoeconomics \& Pharmacoepidemiology, Health Economic Centre, Faculty of Economic and Social Studies, University of Khartoum, Sudan, Email: m_abdalaziz@yahoo.com

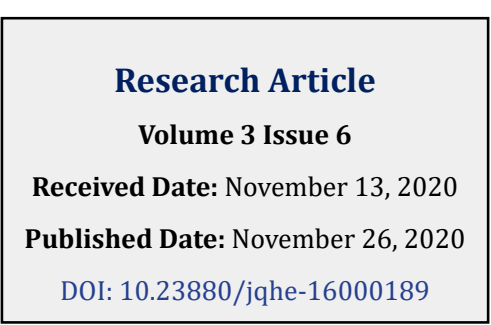

\section{Abstract}

Introduction: HIV/AIDS continues to be a major global health issue, claimed more than 35 million lives so far. In 2017, 1.8 million people becoming newly infected and 940,000 people died from HIV-related causes around the world and approximately 36.9 million people were living with HIV at the end of 2017. Sudan has conducted three rounds of National AIDS spending assessment (NASA). Those rounds was not published or reviewed.

Aims: to systematize review Sudan National AIDS Spending Assessment (NASA) conducted rounds.

Methods: A systematized literature review, qualitative retrospective analytical study. It composed of the current literature that clarifying the research question. SALSA framework was selected and used in this study. Three countries NASA exercise reports China, Iran and Nigeria were selected to be in comparison with Sudan.

Results: Sudan NASA showed that international donors are the main financer for HIV/AIDS services in Sudan. HIV/AIDS spending by Spending Categories (ASC) and beneficiary population revealed that Sudan spent more in programmatic and administration category and non-targeted interventions respectively.

Conclusion: Sudan needs to solve its HIV/AIDS financing paradox to step up funding efficiently and sustainably HIV/AIDS services to end HIV/AIDS epidemic by 2030.

Keywords: Acquired Immunodeficiency Syndrome; Health Expenditures; HIV; Sudan

Abbreviations: NASA: National AIDS Spending Assessment; OECD: Organization for Economic Co-Operation and Development; NHA: National Health Accounts; SHA: System of Health Accounts; MDGs: Millennium Development Goals; SDGs: Sustainable Development Goals; IGAD: Intergovernmental Authority for Development; AIDS: Acquired Immunodeficiency Syndrome; ART: Antiretroviral Therapy; SHO: Sudan Health Observatory; HED: Health Economics Department; BP: Beneficiary Population; HIPCs: Heavily Indebted Poor Countries; PEPFER: President's Emergency Plan for AIDS Relief; SHI: Social Health Insurance; PLHIV: People Living with HIV; MARP: Most at Risk Population.

\section{Introduction}

National AIDS spending assessment (NASA) is a systematic, periodic, multi-sectorial methodology provides a framework and tools for undertaking a comprehensive analysis of actual expenditures related to the national response to HIV/AIDS. Comprehensive and reliable information is essential for health system development and implementation, NASA is a tool that provides continues financial information within the national monitoring and evaluation framework to support better spending in the country. The Organization for Economic Co-operation and Development (OECD) developed The System of Health 
Accounts (SHA 2011) which is an international methodology provides a health expenditure data to track health spending $[1,2]$. National Health Accounts (NHA) is the best source of health expenditure data, which combines expenditure data from all sources and through all types of financial agents. NHA used variations on its theme by including an approach called national account sub-accounts which are estimations exist for specific health areas such as HIV/AIDS [2]. NHA sub-account for HIV/AIDS was the first generation of tools to account for financial resources used for HIV/AIDS health activities. Then, a second adaptation was introduced maintaining the general shape of NHA but mainly made to include HIV/AIDS non-health activities. NASA is the third generation of tools used for all country's HIV/AIDS resource tracking activities [1].

HIV/AIDS continues to be a major global health issue, claimed more than 35 million lives so far. In 2017, 1.8 million people becoming newly infected and 940,000 people died from HIV-related causes around the world and approximately 36.9 million people were living with HIV at the end of 2017 [3]. A call to "halt and reverse" the spread of HIV/ AIDS in areas particularly of high prevalence such as SubSaharan Africa was among the listed goals of the millennium development goals (MDGs) and this call continue to be one of the Sustainable Development Goals (SDGs) sub-targets [4,5]. To be on track towards the end of HIV/AIDS as a global public health threat by 2030, the UN working group on financing for sustainable development estimates that will costs $\$ 36$ billion a year which is twice the current funding level of $\$ 19$ billion [6]. This is indicative of financing challenge ahead and a need to significantly reallocate existing resources to services for HIV/AIDS populations with the highest need and impact [7].

Sudan as officially the Republic of Sudan is a low middleincome country in Northeast Africa occupies a total area of $1,861,484$ square kilometers, making it the third-largest country in Africa [8]. It bordered by seven countries in which HIV/AIDS is highly prevalent [9]. Sudan one of the eight member states of the Intergovernmental Authority for Development (IGAD) that have the largest proportions of cross-border mobile pastoralists and refugees in Africa [10]. The IGAD region represents one of the largest, most complex political, social and economic conditions on the region where population migration and mobility are supposed to be key factors that allow increasing HIV prevalence and incidence rates [11]. Sudan reported the first AIDS case in 1986 and according to UNAIDS 2017, the total number of HIV/AIDS cases in Sudan is 51,000 and the HIV/AIDS prevalence is around $0.2 \%$. This estimation put Sudan among the lowest countries with HIV/AIDS prevalence in Sub-Saharan region but the spread of HIV is influenced by poverty and illiteracy which both are widespread in Sudan $[12,13]$.
HIV is a virus unlike other viruses; the human body can't get rid of it completely, even with treatment and can lead to Acquired Immunodeficiency Syndrome (AIDS) if not managed. So once HIV gets into the body, it will be for life and attacks the body's immune system. HIV can be controlled with antiretroviral therapy (ART) and if it took in a right way, it can prolong the lives of many people infected with HIV, keep them healthy, and lower their chance of infecting others [14]. Despite progress in the fight against HIV/AIDS, there is still no cure and the virus continues to spread, infecting people worldwide. So, countries around the world need to step up funding for HIV/AIDS treatment, prevention, and education to ensure that more people know their status, fewer people contract the fatal illness, and even fewer accidentally pass it on to their partners [15]. NASA has great importance in summarizing the existing financial information to know where the money for HIV/AIDS can be spent more efficiently and equitably [1].

Undertaking a review of Sudan NASA exercise is aim to identify, and summarize the findings of all relevant studies on HIV/AIDS expenditure in Sudan, thereby answering the following question: what are the lessons learned from Sudan National AIDS Spending Assessment rounds? and making the available results more accessible to decision-makers. Among the fourteen review types of literature, the systematized literature review was selected to be conducted for this study. The systematized review is identifying itself parenthetically as a 'systematic' review because it includes one or more elements of the systematic review process while stopping short of claiming that the resultant output is a systematic review. The systematized review is conducted when it's not possible to draw upon the resources required for a full systematic review such as two reviewers or well-defined literature [16,17].

Sudan is a limited resources country provides fund to fight HIV/AIDS through a mix of domestic and external sources. Due to declined fund from external sources recently [18], Sudan needs to make its limited resources go further through tracking them comprehensively. Sudan has conducted three rounds of NASA, first round was covering the fiscal years 2008-2009 [19], second round was covering the fiscal years 2011-2012-2013 [20] and the third round was covering the fiscal year 2014 [21]. Those rounds had not been reviewed before to show if the provided indicators by NASA exercise are on the track towards the end of the HIV/ AIDS epidemic as one of SDG3 targets or not [7]. Conducting a systematized review of Sudan NASA is important because many surveys were done before but not published or reviewed, through this study, the pattern of Sudan HIV/ AIDS spending will be displayed helping stakeholders to take a strategic decision towards sustainable health financing policies and to have a well-functioning health system meets 
the needs of people living with HIV/AIDS and people at-risk which is crucial to ending HIV/AIDS epidemic by 2030. The study was focused into three categorical points: the spending on HIV/AIDS in Sudan by international entities, the pattern of spending on HIV/AIDS in Sudan and a comparison between HIV/AIDS spending pattern in Sudan and other countries HIV/AIDS spending pattern. The objective of this paper is to systematize review the Sudan National AIDS Spending Assessment (NASA) rounds.

\section{Materials and Methods}

A systematized qualitative retrospective exploratory analytical study accompanied by the comparative descriptive study. The study conducted on Health Economics Department at the Federal Ministry of Health in Khartoum State. The study population was composed of the current literature that clarifying the research question.

Non-probability sampling, purposeful sampling for the comparative study. purposeful sampling only targeting a specific population that appear to be representative for the comparison between HIV/AIDS spending pattern in Sudan and other countries conducting NASA exercise in the same period. This study used SALSA framework: the Search, Appraisal, Synthesis, and Analysis framework which is a simple framework divides the review process into four main stages: (1) search (2) appraisal (3) synthesis and (4) analysis.

\section{Criteria Used to Consider Reviews for Inclusion}

Inclusion and exclusion criteria were specified in the prior study to perform the literature search, and the studies were selected based on these criteria. Those criteria ensure that studies are selected systematically and systematized reviews do possess a greater likelihood of bias than systematic reviews which adhere more strictly to guidelines because the systematized review is typically conducted as a postgraduate student assignment and completion of the academic requirements for the review is prioritized over methodological considerations [16]. The criteria for inclusion were that studies:

- Included all the search terms in the title or the abstract to ensure focus.

- Could be obtained in full-text to ensure availability.

- Could be published or unpublished but not more than 10 years ago.

- Country of comparison should conduct NASA exercise in the same period or maximum one year difference from the time that Sudan NASA exercise was conducted in.

- Country NASA report should be in English.

- Country NASA report is published online

- Country NASA report is available in full text.
Three countries NASA exercise reports was selected to be in comparison with Sudan NASA 2009, 2012 and 2014 reports [19-21], those countries were China NASA 2010, Iran NASA 2012 and Nigeria NASA 2014 [22-24]. The criteria for exclusion were that studies were not published in English.

\section{Search Methods for the Identification of Reviews}

Search stage: To design a search strategy, the research question had broken down into different aspects of each aspect analyzed for possible synonyms, abbreviations and alternative spellings. we used the following search terms Sudan, National AIDSSpendingAssessmentand NASA Exercise as a synonymous for National AIDS Spending Assessment, the complex search strings were created using OR, AND operator [25]. The following search string was generated and applied to the search in all databases mentioned previously for the period from August, 2018 to Jan 2019: "National AIDS Spending Assessment" AND "Sudan", "NASA Exercise" AND "Sudan", "National AIDS Spending Assessment" OR "NASA Exercise" AND "Sudan". In The Cochrane Library, PubMed and Google scholar the search string was applied using the advanced search. In Sudan Health Observatory we searched in reports using health economics as a theme and we used "National AIDS Spending Assessment" as searching term because this site is generated by Sudan Federal Ministry of Health to provide information and knowledge in support of Sudan evidence-based health policymaking and planning. We used the search string to search in reports prepared by the Health Economics Department at the Federal Ministry of Health in Khartoum State.

The search stage in a systematized review takes the most easily identified elements of systematicity (16). It involves collecting information in one or more databases according to search strategy which is relevant for the chosen research question. This stage aims to define the research list of studies to be analyzed.

Information sources:

\section{All published studies and grey literature were identified by searching the following:}

- Four electronic databases: The Cochrane Library, PubMed, Google scholar, Sudan Health Observatory.

- Manually in the reports prepared by the Health Economics Department at the Federal Ministry of Health in Khartoum State [26].

The last search was run on the 26th of January 2019.

For the comparative issue, data collected through documents review of China NASA 2010, Iran NASA 2012 and Nigeria NASA 2014 using a data extraction form adapted from 
a data extraction form generated by Cochrane collaboration (see Supplementary data). The comparison was between Sudan NASA 2009, 2012 and 2014 reports and China NASA 2010, Iran NASA 2012 and Nigeria NASA 2014 against certain criteria to clarify the specific objective which is to compare between HIV/AIDS spending pattern in Sudan and other countries HIV/AIDS spending pattern. Those criteria were:

- HIV/AIDS Spending by Financing Sources.

- HIV/AIDS Spending by ASC (care and treatment, prevention and Program Management and Administration).

- HIV/AIDS Spending by BP (PLHIV, MARP and NonTargeted Interventions).

\section{Assessment of the Methodological Quality of the Included Reviews}

Appraisal stage: After we collected the studies, we applied the exclusion and inclusion criteria to select studies to review. In a systematic review, the quality of studies should be assessed and that helped to assess and evaluate the inclusion and exclusion criteria, guide the interpretation of findings and provide recommendations for further research, but in this systematized review the quality assessment was not identifiable because we used a small set of eligible studies [16]. The study selection process was summarized in a flow diagram shown in the results chapter.

\section{Data Extraction and Management}

Data was collected through a systematic approach to review the current literature which answers the research question and fulfil the study objectives. The systematized literature review used SALSA framework: the Search, Appraisal, Synthesis, and Analysis framework which is a simple framework adapted to review Sudan NASA and it has been applied to characterize the systematized review. The SALSA framework divides the review process into four main stages: (1) search (2) appraisal (3) synthesis and (4) analysis [25]. Analysis stage: We analyzed the extracted data manually, using thematic analysis which is "a method for identifying, analyzing and reporting patterns within data", thematic analysis is an initial and simple method of analysis. The goal of thematic analysis is to identify themes, which are patterns in the relevant and important data, then use these themes to address the research question. The thematic analysis not about summarizing data, it interprets and makes sense of them $[27,28]$. NASA exercise is a quantitative methodology and thematic analysis is used for the analysis of qualitative methodology. To use thematic analysis, we attribute a qualitative thematic description to all quantitative data [29] in NASA exercise by using variables in NASA exercise as codes [30] and build up themes out of this codes, variables are the key findings (i.e. indicators) in NASA exercise, Indicators defined as an observed value of variables fields [31]. All studies included are similar. So, we derived key themes using NASA functional framework that analyzes data more convenient to answer the research question. The aggregated data was displayed using bar graphs which are the only way with pie chat to visualize qualitative data [32].

Data extracted were synthesized and presented tabulated with highlighting the differences between studies.

\section{Data Synthesis}

Synthesis stage: Synthesis of data is the next stage after identifying studies included in this systematized review and it aims to create a summary of findings which are previously defined in the included study [33]. It initialized with the data extraction phase and done in a qualitative manner [25]. To extract data, data extraction forms should be designed to obtain information vital to the research question from the included studies. We designed a data extraction form adapted from a data extraction form generated by Cochrane collaboration (see Supplementary data), the form includes general information about the study and data relevant to research objectives which answer the research question, we extracted data manually and describe them as stated in reports. We used the following strategy to extract data that we extracted data from each study separately, and then we collated, summarized and tabulated them. Another strategy can be approached by extracting data from all studies directly into a single data extraction form but we selected the first one because the studies are detailed with the same methodology and relating to different periods of times [25,34].

\section{Results}

\section{Description of the Included Reviews}

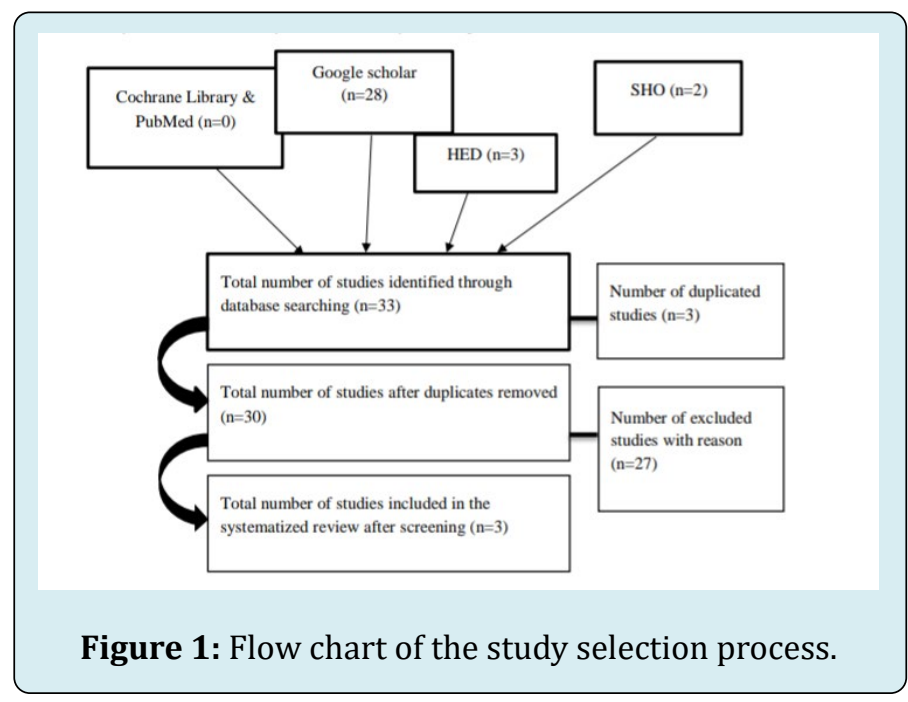


Figure 1 shows the studies that were included in the systematized review. Out of 33 studies, only 3 studies met the inclusion criteria and were included in the review. The studies included were from Sudan. The search resulted in $\mathrm{n}=33$ studies, searching in The Cochrane Library, PubMed resulted in $\mathrm{n}=0$ study, searching in Google scholar using the following search strings "National AIDS Spending Assessment" AND "Sudan", "NASA Exercise" AND "Sudan", "National AIDS Spending Assessment" OR "NASA Exercise" AND "Sudan" resulted in $\mathrm{n}=28$ studies. Searching in Google scholar using the search string "NASA Exercise" AND "Sudan" resulted in $\mathrm{n}=0$ study. searching in Sudan Health Observatory (SHO) website resulted in $\mathrm{n}=2$ studies $\mathrm{s}$ and searching among reports prepared by Health Economics Department (HED) at Federal Ministry of Health in Khartoum State resulted in n= 3 studies A total of three duplicates were removed manually. After duplicates were removed, 30 studies were screened; the screening process removed 27 studies (26 studies because of the inclusion criteria, and 1 study because of the exclusion criteria). The remaining three studies were synthesized then analyzed thematically. Twenty-six studies were removed because they did not meet the inclusion criteria.

- 26 studies, from Google Scholar, were removed because the search terms were not included in title or abstract and four studies from the twenty-six studies, were not available in a full text also. One study was removed because of the exclusion criteria:

- A study from Google Scholar has removed because of it a non-English study.

\section{Characteristics and Methodological Aspects of the Included Reviews}

All three NASA rounds published as a full report using NASA methodology as study design. First round conducted in 2011 covering the fiscal years 2008-2009, second round conducted in 2015 covering the fiscal years 2011-2012-2013 and the third round was conducted in 2016 covering the fiscal year 2014.

\section{HIV/AIDS Spending Pattern In Countries Conducting NASA Exercise}

\begin{tabular}{|c|c|c|c|c|c|}
\hline \multirow{2}{*}{ Main Indicators } & \multirow{2}{*}{ Indicators by category } & \multicolumn{4}{|c|}{ Percentages of total HIV spending } \\
\hline & & China & Nigeria & Iran & Sudan* \\
\hline \multirow{3}{*}{$\begin{array}{l}\text { HIV/AIDS Spending by Financing } \\
\text { Sources }\end{array}$} & By Public Source & $92.70 \%$ & $27.07 \%$ & $57 \%$ & $22.14 \%$ \\
\hline & By International Source & $6.80 \%$ & $70.81 \%$ & $8 \%$ & $74 \%$ \\
\hline & Private Source & $0.50 \%$ & $2.12 \%$ & $35 \%$ & $4.62 \%$ \\
\hline \multirow{3}{*}{$\begin{array}{l}\text { HIV/AIDS Spending by AIDS Spending } \\
\text { Categories (ASC) }\end{array}$} & Care and Treatment & $52.70 \%$ & $30.17 \%$ & $9.60 \%$ & $8.80 \%$ \\
\hline & $\begin{array}{l}\text { Program Management and } \\
\text { Administration }\end{array}$ & $24.50 \%$ & $13.62 \%$ & $5.80 \%$ & $48.70 \%$ \\
\hline & Prevention & $19.80 \%$ & $25.62 \%$ & $81.10 \%$ & $26.30 \%$ \\
\hline \multirow{3}{*}{$\begin{array}{l}\text { HIV/AIDS Spending by beneficiary } \\
\text { population ( BP) }\end{array}$} & People living with (PLHIV)HIV & $57.57 \%$ & $32.76 \%$ & $11.20 \%$ & $12.33 \%$ \\
\hline & Most at risk (MARP)population & $7.99 \%$ & $2.22 \%$ & $45.20 \%$ & $5.42 \%$ \\
\hline & Non-Targeted Interventions & $23.59 \%$ & $36.85 \%$ & $8.20 \%$ & $52.06 \%$ \\
\hline
\end{tabular}

*Average Sudan NASA, 2009; 2012 and 2014.

Table 1: Summary of HIV/AIDS Spending Pattern in Countries Conducting NASA Exercise.

Table 1 illustrated Spending Pattern in Countries Conducting NASA Exercise by Financing Sources, Categories (ASC) and by beneficiary population (BP).

\section{Discussion}

Through all NASA rounds conducted in Sudan, international donors were the main financers of HIV/ AIDS services followed by public sector as financing source for HIV/AIDS services. HIV/AIDS financing by private sector showed a minimal spending share of total HIV/AIDS expenditure. Conversely, literature showed that Sudan public health expenditure is $28.87 \%$ and $22.34 \%$ of total health expenditure and international health expenditure (donors) is $4.16 \%$ and $4.52 \%$ of total health expenditure for the year 2008 and 2011 respectively. Also, NHA revealed that Sudan has a low financial protection for health, the household outof-pocket spending $66.97,69.88 \%$ and $76 \%$ of total health spending as estimated 2008, 2011 and 2013 respectively [35]. Jose Izazola [36], UNAIDS 2018, showed that, historically, the HIV response has been largely funded by international donors which is declined recently due to a number of factors including the depreciation of donor currencies and delays in funding from the USA (the biggest donor). To be on course to end HIV/AIDS as a public health threat by 2030, UNAIDS estimates that US $\$ 26.2$ billion will be required for the global 
HIV response in 2020. In order to reach the 2020 target, the world must increase the amount of resources available for the HIV and as a result, there is an emphasis on countries most affected by the HIV epidemic to finance their own responses and find more efficient and cost-effective ways to do so. Recently, most of low- and middle-income countries are beginning to lead on efforts to finance their HIV response and shifting towards domestic funding to fill some funding gaps and working towards more sustainable response to HIV/AIDS. Also literature showed, Sudan is one of the Heavily Indebted Poor Countries (HIPCs) with an external debt more than 37 billion US\$ and according to the World Bank, this debt is unsustainable as Sudan is unable to service it while struggling to reduce poverty and reaching the developmental goals at the same time [35].

Sudan NASA rounds revealed that Sudan is highly donor's dependent country and at the same time Sudan has a low share by donors of total health expenditure. However, Sudan is one of the Heavily Indebted Poor Countries needs to step up funding their own responses to fight AIDS as a public health threat. This paradox faces Sudan and it needs new ideas to help Sudan to be on the track to end HIV/AIDS by 2030 .

Jose Izazola [36] presented Vietnam Transitional funding case, that spending on HIV in Vietnam was estimated at US\$137.5 million. The Global Fund to Fight AIDS, Tuberculosis and Malaria (Global Fund) funded 36\% funded by The USA's President's Emergency Plan for AIDS Relief (PEPFAR) while $14 \%$ As a result, the vast majority of people living with HIV received free antiretroviral treatment in 2015. As the country moves from low-income to a lower middleincome status, International funds for HIV programme in Vietnam were decreased. In response, the Vietnamese government has invested in finding more sustainable way to ensure HIV treatment and has committed to covering $70 \%$ of treatment needs by 2018 at a cost of US\$15-20 million for first-line antiretroviral therapy (ART). In order to fund this, the government has centralized ARV procurement, and is funding costs through its Social Health Insurance (SHI) fund. The WHO report 2014 [35], showed that health financing system in Sudan is weak and fragmented, in spite of the many reforms which had been made to it such as introducing health insurance and free of charge care policies. Sudan can adopt Vietnam transitional funding case to increase the public share for HIV/AIDS services but the health financing system in Sudan needs effective governance and proper management to reflect positively on rise financing by public sector for HIV/AIDS services.

All Sudan NASA rounds showed that, the pattern of spending on HIV/AIDS activities were mainly in programme management and administration category followed by prevention, care and treatment categories. As well, the pattern of spending on HIV/AIDS beneficiary population were non-targeted interventions which include national systems strengthening and programme management groups and not targeted any person or population. Spending on people living with HIV/AIDS and most at risk populations witnessed a remarkable increasing pattern through all Sudan NASA rounds which started with minimal spending in the first round and increased gradually in the second and third NASA rounds. HIV allocative efficiency study done by world bank [37] suggested that in a limited resources country as Sudan, more HIV funding should be allocated to four priority high-impact programs: ART, prevention among FSW, prevention among men at higher risk (clients of sex workers) and MSM. The World Bank optimization analyses revealed that if the programmatic spending levels and allocations of the financial year 2013 continue in Sudan as it is, the HIV incidence rate is expected to rise, especially in FSW, MSM and clients and If the programmatic expenditure in 2013 was held constant, increased HIV incidence rates, combined with the population growth rate of about $1.5 \%$, this will lead to significant increases in the number of new HIV infections in all population groups in the medium term. Accordingly, Sudan needs to focus in the four priority highimpact programs discussed by World Bank report, which are care and treatment, prevention categories and most at-risk populations to decrease HIV/AIDS incidence which help to fight HIV/AIDS as a public health threat by 2030 .

Comparison between average Sudan HIV/AIDS pattern of expenditure and HIV/AIDS pattern of expenditure of other countries conducted NASA exercise on the same period of time revealed that China and Iran public financing sources had the great share of total HIV/AIDS expenditure. Conversely, Sudan and Nigeria had the great share of total HIV/AIDS expenditure by international financing sources. China and Nigeria showed that spending on Care and Treatment category is the main area of concern followed by prevention category in Nigeria and Program Management and Administration category in China. The main area of concern on HIV/AIDS spending by ASC in Iran was prevention category. On the other hand, Sudan revealed that Program Management and Administration category had the great share of total HIV/ AIDS spending by ASC followed by prevention and Care and Treatment categories respectively. HIV/AIDS spending by beneficiary group in China was mainly by People living with HIV (PLHIV) and in Iran was dominate by Most at risk population (MARP). Conversely, Non-Targeted Interventions were the dominant beneficiary group that spending more funds of total HIV/AIDS expenditure in Sudan and Nigeria.

This comparison shows that countries as China and Iran financing their HIV/AIDS services mainly by the public sectors, they are investing more in the high priority areas 
such as care and treatment, prevention, PLHIV and MARP to offer a sustainable response to fight HIV/AIDS epidemic. On the other hand, countries such as Nigeria and Sudan financing their HIV/AIDS services mainly by international sources, they are investing mainly in categories such as Non-Targeted Interventions and Program Management and Administration which is against the recommended areas of investment discussed by the World Bank allocative efficiency report [37]. Finally, if this pattern of spending continues in Sudan as it is, the HIV incidence rate is expected to rise and Sudan will face unsustainable response to fight HIV/AIDS epidemic.

\section{Conclusions}

This systematized review shows that Sudan as one of LIMICs with low-level concentrated HIV epidemic is highly dependent on international donors funds which affect the sustainability of country response to fight HIV/AIDS epidemic and make HIV/AIDS continue as a public health threat. Accordingly, Sudan HIV/AIDS financing pattern is unsustainable, it needs reform because Sudan total health expenditure had a low share by international donors which indicate that Sudan can increase spending on HIV/AIDS by public financing source through effective governance and proper management. Also, this study reveals that Sudan HIV/ AIDS spending pattern focus in Non-Targeted Interventions as beneficiary population and in Program Management and Administration category. It needs to focus on the four priority high-impact programs discussed by World Bank report, which are care and treatment, prevention categories and most at-risk populations to decrease HIV/AIDS incidence which help to fight HIV/AIDS as a public health threat by 2030. Countries with high domestic funding for HIV/AIDS services are investing efficiently on the priority high-impact programs as care and treatment, prevention categories and most at-risk populations and they are offering a sustainable response to fight HIV/AIDS epidemic. Sudan needs to solve its HIV/AIDS financing paradox to step up funding efficiently and sustainably HIV/AIDS services to end the HIV/AIDS epidemic by 2030 .

\section{Policy Implications}

\section{This review recommended the following:}

1. To review the dependency on external funds by concerned partners, and to share the experience of China and Iran in financing HIV/AIDS services by public sectors as the main financer.

2. To Increase spending on prevention and care and treatment categories.

3. Sudan should consider spending a higher share on most at-risk populations to decrease the incidence of HIV/ AIDS among the general population and to fight HIV/
AIDS epidemic by 2030.

4. To readdress spending on programmatic and administration category.

5. To conduct a new NASA exploring the recent country response to fight HIV/AIDS.

\section{Authors' Contributions}

SK and KHH conceived the study; SK and kHH designed the study protocol; BJA and HGM carried out the clinical assessment; $\mathrm{SK}, \mathrm{KHH}, \mathrm{MM}$ carried out the analysis and interpretation of these data. SK, KHH and MM drafted the manuscript; SK, KHH and MM critically revised the manuscript for intellectual content. All authors read and approved the final manuscript. MM and $\mathrm{KHH}$ are guarantors of the paper.

\section{Acknowledgements}

The authors would like to acknowledge that this paper was part of a thesis conducted at University of Medical Sciences \& Technology, Khartoum, Sudan.

The author(s) received no financial support for the research, authorship, and/or publication of this article.

\section{Ethical Approval}

Ethical clearance was obtained from the Institutional Review Board of University of Medical Sciences and Technology and the Research and Ethical Committee at Khartoum State Ministry of Health-Research Department.

\section{Financial Resources}

The authors would like to acknowledge that this paper was part of a thesis conducted at University of Medical Sciences \& Technology, Khartoum, Sudan.

\section{References}

1. (2009) Joint United Nations Programme on HIV/AIDS. Guide to produce National AIDS Spending Assessment (NASA), UNAIDS, pp: 1-84.

2. (2010) World Health Organization, editor. Monitoring the building blocks of health systems: a handbook of indicators and their measurement strategies. Geneva: World Health Organization, USA pp: 92.

3. (2020) HIV/AIDS. World Health Organization.

4. (2020) Physician AB-C. Are We on Track to Reverse Global HIV?. Verywell Health. 
5. (2020) SDG 3: Ensure healthy lives and promote wellbeing for all at all ages. SDG Compass.

6. Oberth G (2015) Financing the Global Goals: Why the Global Fund replenishment matters for the SDGs. Aidspan 272.

7. (2020) Resources and financing for the AIDS response.

8. (2019) Sudan. Wikipedia.

9. Health in Sudan-Wikipedia.

10. Serbessa MK, Mariam DH, Kassa A, Alwan F, Kloos H (2016) HIV/AIDS among pastoralists and refugees in north-east Africa: a neglected problem. African Journal of AIDS Research 15(1): 45-54.

11. IGAD (2010) IGAD regional HIV/AIDS partnership program adopts cross border treatment for HIV/AIDS patients in the region.

12. UNAIDS (2017) Country factsheets Sudan 2017.

13. Mohamed BA, Mahfouz MS (2013) Factors Associated with HIV/AIDS in Sudan. Biomed Res Int 2013: 971203.

14. (2017) What Are HIV and AIDS?.

15. (2017) 5 Reasons Why We Need to Keep Funding the Fight Against HIV/AIDS. Global Citizen.

16. Grant MJ, Booth A (2009) A typology of reviews: an analysis of 14 review types and associated methodologies. Health Information \& Libraries Journal 26(2): 91-108.

17. Tranfield D, Denyer D, Smart P (2003) Towards a Methodology for Developing Evidence-Informed Management Knowledge by Means of Systematic Review. British Journal of Management 14(3): 207-222.

18. WHO (2020) Health Accounts Methodology and Tools. WHO.

19. Mustafa DM (2020) Health Economics DepartmentFederal Ministry of Health Sudan NASA 2008-2009.

20. Mustafa DM. Sudan NASA 2011-2013.

21. Mustafa DM. Sudan NASA 2014. 2014.

22. Shan D, Sun J, Yakusik A, Chen Z, Yuan J, et al. (2013) Total
HIV/AIDS Expenditures in Dehong Prefecture, Yunnan Province in 2010: The First Systematic Evaluation of Both Health and Non-Health Related HIV/AIDS Expenditures in China. PLOS ONE 8(6).

23. Aboulhallaje M, Najafi B (2012) Iran National AIDS Spending Assessment (NASA). pp: 1-65.

24. Idoko J (2015) Nigeria_NASA_2013-2014. National Agency for the Control of AIDS.

25. Puks R, Smorgun I, Lamas D (2016) Software Tools for Supporting Literature Reviews: An Overview and a Case Study. pp: 82.

26. Rickman K (2020) Research Tips: Systematic Reviews Step-by-Step.

27. Maguire M, Delahunt B (2017) Doing a Thematic Analysis: A Practical, Step-by-Step Guide for Learning and Teaching Scholars. All Ireland Journal of Higher Education 9(3): 3351-33514.

28. Braun, Clarke (2006) What is Thematic Analysis?. How to Use Thematic Analysis. pp: 79.

29. The Joanna Briggs Institute (2014) Methodology for JBI Mixed Methods Systematic Reviews,The Joanna Briggs Institute Reviewers' Manual.

30. Morgan D (2020) Can I use thematic analysis for quantitative studies?.

31. Babbie E (2012) The Practice of Social Research. In: $14^{\text {th }}$ (Edn.), Cengage Learning.

32. (2016) Display of qualitative data. Statistics Made Easy.

33. Saragossi J (2020) Systematic Reviews: Data Extraction. Research \& Subject Guides.

34. Higgins J, Green S (2011) Cochrane Handbook for Systematic Reviews of Interventions.

35. (2016) Public Health Institute. Health Financing System Review Report May 2014.

36. Izazola J (2015) Funding for HIV and AIDS. AVERT.

37. (2014) University of New South Wales. Sudan's HIV response: Value for money in a low-level HIV epidemic. Findings from the HIV allocative efficiency study. 